\title{
Biomimetic Structures on Steel via Self-organization Processes in Multiple- scan, fs-laser irradiated surfaces
}

\author{
Camilo Florian $^{1 \dagger}$, Daniel Puerto ${ }^{1}$, Yasser Fuentes-Edfuf ${ }^{1}$, Evangelos Skoulas ${ }^{2}$, Emmanuel Stratakis ${ }^{2}$, \\ Javier Solis $^{1^{*}}$ and Jan Siegel ${ }^{1+\dagger}$
}

1. Laser Processing Group, Instituto de Óptica, CSIC, Serrano 121, 28006 Madrid, Spain
2. Institute of Electronic Structure and Laser (IESL), Foundation for Research and Technology (FORTH), N. Plastira 100, Vassilika
Vouton, 70013, Heraklion, Crete, Greece

† camilo.florian@io.cfmac.csic, †† j.siegel@csic.es.*Presenting author: j.solis@io.cfmac.csic.es

Weakly focused fs-pulses have the potential to produce nanometer structures that self-organize under certain irradiation conditions [1]. Such structures, known as LIPSS [2] (laser induced periodic surface structures), are potential candidates for mimicking surface textures found in nature, on materials of technological interest. This enables surface functionalization for a variety of applications including wettability, optical response, and wear resistance $[3,4]$.

In this work, different laser-generated surface structures resembling the skin of certain reptiles have been synthesized upon multi-scan laser irradiation of stainless steel. For the fabrication of the structures we used a high-repetition rate femtosecond laser $(1030 \mathrm{~nm}, 350 \mathrm{fs}$, up to $2 \mathrm{MHz})$ and a galvo-scanner combined with a Ftheta lens, allowing large-area processing. Different structures are generated, depending on the laser processing parameters (scan speed, polarization, laser fluence and total number of scans). Their morphological analysis reveals that their feature size and geometry strongly depends on the irradiation parameters as shown in Fig.1 where the impact of the multi-scan approach can easily be appreciated (notice the scale difference of the images). This provides and additional control parameter to generate novel functional structures.
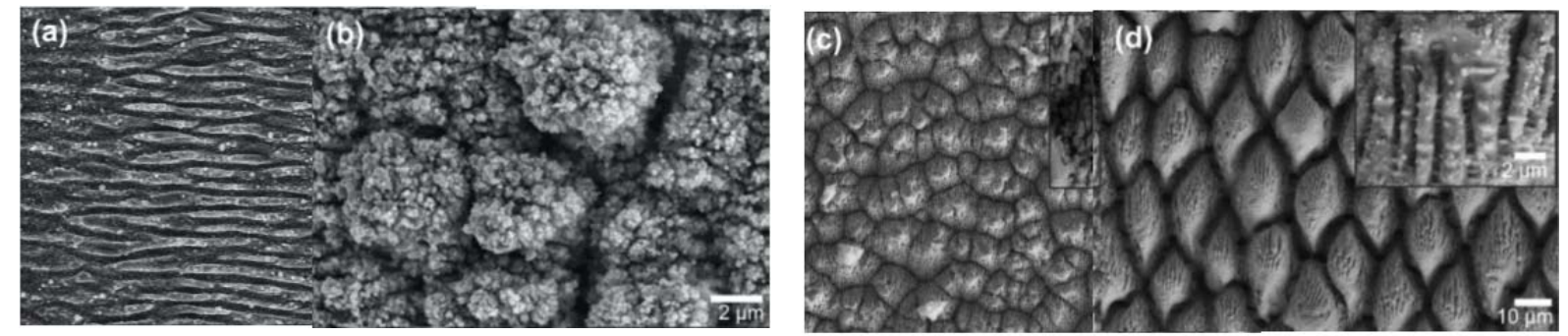

Fig. 1 Scanning Electron Microscopy images of surface structures fabricated on steel. (a) and (b) show structures that were produced after 1 laser scan at of $0.38 \mathrm{~J} / \mathrm{cm}^{2}$ and $2 \mathrm{~J} / \mathrm{cm}^{2}$ respectively, while (c) and (d) were produced with 1200 and 200 over-scans with fluences of $0.2 \mathrm{~J} / \mathrm{cm}^{2}$ and $1 \mathrm{~J} / \mathrm{cm}^{2}$ respectively.

Measurements performed on the structures with water and oil show that the wetting properties induced are also strongly dependent on the morphology of the generated structures. The strategy here presented provides new perspectives for increasing fluid transport, improving biocompatibility, and tuning tribological properties of materials.

\section{References}

[1] G. D. Tsibidis, C. Fotakis, and E. Stratakis "From ripples to spikes: a hydrodynamical mechanism to interpret femtosecond laser-induced self-assembled structures" Phys. Rev. B 92041405 (2015)

[2] J. F. Young, J. S. Preston, H. M. van Driel, and J. E. Sipe, "Laser-induced periodic surface structure. II. Experiments on Ge, Si, Al, and brass," Phys. Rev. B 27, 1155 (1983)

[3] C. Greiner and M. Schäfer "Bio-inspired scale-like surface textures and their tribological properties" Bioinspir. Biomim. 10044001 (2015)

[4] D. H. Kam, S. Bhattacharya and J. Mazumder "Control of the wetting properties of an AISI 316L stainless steel surface by femtosecond laser induced surface modification” J. Micromech. Microeng. 22, 105019 (2012). 